\title{
Spatio-temporal credit assignment in neuronal population
} learning.

\section{Text S1: Relating the plasticity rule to a gradient ascent procedure}

Johannes Friedrich ${ }^{1}$, Robert Urbanczik ${ }^{2}$, Walter Senn ${ }^{3, *}$

1,2,3 Department of Physiology, University of Bern, Bühlplatz 5, CH-3012 Bern, Switzerland

* E-mail: senn@pyl.unibe.ch

We show how the plasticity rule presented in the main text is based on a gradient ascent procedure maximizing the average reward rate. This Supplementary Material is organized as follows: First, a formula is derived for the gradient, with respect to synaptic strength of the population neurons, for the probability of taking a behavioral decision. We next summarize Baxter and Bartlett's framework (Ref. [1] main text) for learning in partially observable Markov decision processes (POMDP's). Finally, we show how our population gradient leads to a learning rule for POMDP's and how this procedure, formulated in discrete time, transforms to the simplified online plasticity rule presented in the main text.

\section{Gradient for the behavioral decision}

Let $\mathbf{X}$ be the spike pattern presented to the population neurons and $\mathbf{W}$ the matrix of their synaptic strength. The probability $P_{\mathbf{W}}(D \mid \mathbf{X})$ of responding with decision $D$ to the stimulus is

$$
P_{\mathbf{W}}(D \mid \mathbf{X})=\int \mathrm{d} \mathbf{Y} P(D \mid A(\mathbf{Y})) \prod_{\nu=1}^{N} P_{\mathbf{W}^{\nu}}\left(Y^{\nu} \mid \mathbf{X}^{\nu}\right) .
$$

Here, the conditional probability of the decision, $P(D \mid A)$, is given by Eq. (9, main text), the definition of the activity $A(\mathbf{Y})$ is given just above Eq. (9, main text). The probability $P_{\mathbf{W}^{\nu}}\left(Y^{\nu} \mid \mathbf{X}^{\nu}\right)$ that neuron $\nu$ produces the postsynaptic spike train $Y^{\nu}$ in response to its version $\mathbf{X}^{\nu}$ of the stimulus is obtained by applying Eq. (7, main text) to neuron $\nu$.

We will only need the gradient of $P_{\mathbf{W}}(D \mid X)$ for a single stimulus. So, ignoring the dependence on $X$, we write

$$
P_{\mathbf{W}}(D)=\int \mathrm{d} \mathbf{Y} P(D \mid A(\mathbf{Y})) \prod_{\nu=1}^{N} P_{\mathbf{W}^{\nu}}\left(Y^{\nu}\right) .
$$

To lighten the notation further, we focus on calculating the gradient of $P_{\mathbf{W}}(D)$ only with respect to the strength of one of the synapses (the expressions for the other synapses being entirely analogous). Let $w$ denote the strength of the first synapse of the first population neuron and let $Y=Y^{1}$ the postsynaptic spike train produced by this neuron. To isolate the contribution of the first neuron we decompose the activity $A(\mathbf{Y})$ as

$$
A(\mathbf{Y})=\frac{1}{\sqrt{N}} c(Y)+A^{\backslash}\left(Y^{2}, \ldots, Y^{N}\right) \quad \text { with } A^{\backslash}=\frac{1}{\sqrt{N}} \sum_{\nu=2}^{N} c\left(Y^{\nu}\right)
$$

As random variables, $Y$ and $A^{\backslash}$ are independent, since the probability density on the postsynaptic spike trains $\mathbf{Y}$ in Eq. (1) is given by a product. Further the density on $Y^{2}, \ldots, Y^{N}$ induces a density $\mu$ on $A \backslash$, crucially $\mu$ does not depend on $w$. With this notation, we can rewrite (1) as

$$
P_{w}(D)=\int \mathrm{d} Y \mathrm{~d} A^{\backslash} \mu\left(A^{\backslash}\right) P\left(D \mid \frac{c(Y)}{\sqrt{N}}+A^{\backslash}\right) P_{w}(Y) .
$$


We now set

$$
\begin{aligned}
\mathrm{S} P\left(D \mid A^{\backslash}\right) & =\frac{1}{2}\left(P\left(D \mid \frac{1}{\sqrt{N}}+A^{\backslash}\right)+P\left(D \mid \frac{-1}{\sqrt{N}}+A^{\backslash}\right)\right) \\
\mathrm{DP}\left(D \mid A^{\backslash}\right) & =\frac{1}{2}\left(P\left(D \mid \frac{1}{\sqrt{N}}+A^{\backslash}\right)-P\left(D \mid \frac{-1}{\sqrt{N}}+A^{\backslash}\right)\right)
\end{aligned}
$$

and have

$$
P\left(D \mid \frac{c(Y)}{\sqrt{N}}+A^{\backslash}\right)=\mathrm{S} P\left(D \mid A^{\backslash}\right)+\mathrm{DP}\left(D \mid A^{\backslash}\right) c(Y) .
$$

Plugging this into the above expression for $P_{w}(D)$ we obtain

$$
P_{w}(D)=\int \mathrm{d} A^{\backslash} \mu\left(A^{\backslash}\right) \mathrm{S} P\left(D \mid A^{\backslash}\right)+\int \mathrm{d} Y \mathrm{~d} A^{\backslash} \mu\left(A^{\backslash}\right) \mathrm{DP}\left(D \mid A^{\backslash}\right) c(Y) P_{w}(Y) .
$$

Now, since the first integral does not depend on $w$, we have

$$
\frac{\partial}{\partial w} P_{w}(D)=\int \mathrm{d} Y \mathrm{~d} A^{\backslash} \mu\left(A^{\backslash}\right) \operatorname{DP}\left(D \mid A^{\backslash}\right) c(Y) \frac{\partial}{\partial w} P_{w}(Y) .
$$

To bring this result into a form which is usable in the Monte Carlo sampling procedure below, we first rewrite it as

$$
\begin{aligned}
\frac{\partial}{\partial w} P_{w}(D) & =\int \mathrm{d} Y \mathrm{~d} A^{\backslash} \mu\left(A^{\backslash}\right) \mathrm{D} P\left(D \mid A^{\backslash}\right) c(Y) \frac{\partial}{\partial w} P_{w}(Y) \\
& =\int \mathrm{d} Y \mathrm{~d} A^{\backslash} \mu\left(A^{\backslash}\right) P(D \mid A) \frac{\mathrm{D} P\left(D \mid A^{\backslash}\right)}{P(D \mid A)} c(Y) P_{w}(Y) \frac{\frac{\partial}{\partial w} P_{w}(Y)}{P_{w}(Y)} \\
& =\int \mathrm{d} Y \mathrm{~d} A^{\backslash} \mu\left(A^{\backslash}\right) P_{w}(Y) P(D \mid A) \frac{\mathrm{D} P\left(D \mid A^{\backslash}\right)}{P(D \mid A)} c(Y) \frac{\partial}{\partial w} \ln P_{w}(Y),
\end{aligned}
$$

where from the second line on we have used $A$ as shorthand for $A=\frac{1}{\sqrt{N}} c(Y)+A \backslash$. The product of densities the third line, $\mu\left(A^{\backslash}\right) P_{w}(Y) P(D \mid A)$, is just the joint density, $\mu\left(A^{\backslash}\right) P_{w}(Y) P(D \mid A)=P_{w}(D, A \backslash, Y)$. For the Monte Carlo procedure we decompose the joint density as $P_{w}(D, A \backslash, Y)=P_{w}(D) P_{w}(A \backslash, Y \mid D)$ to obtain our final expression for the gradient

$$
\frac{\partial}{\partial w} P_{w}(D)=P_{w}(D) \int \mathrm{d} Y \mathrm{~d} A^{\backslash} P_{w}\left(A^{\backslash}, Y \mid D\right) \frac{\mathrm{D} P\left(D \mid A^{\backslash}\right)}{P(D \mid A)} c(Y) \frac{\partial}{\partial w} \ln P_{w}(Y)
$$

\section{Policy gradient learning in a POMPD}

A partially observable Markov decision problem involves a finite number of states which we shall denote by lower case bold symbols such as $\mathbf{i}$ or $\mathbf{j}$. Each state is associated with a reward value $R(\mathbf{i})$ which may depend deterministically or stochastically on $\mathbf{i}$. The behavior of the learning agent iteratively generates a discrete time Markov process as follows. Assuming the process is in state $\mathbf{i}$ at discrete time step $n-1$, then:

- The agent makes an observation $\mathbf{X}(\mathbf{i})$, which may be partial and noisy, so it may be impossible to uniquely identify the underlying state $\mathbf{i}$ based on $\mathbf{X}(\mathbf{i})$. As the notation suggests, $\mathbf{X}(\mathbf{i})$ corresponds to the stimulus presented to our decision network.

- Based on the observation, the agent generates a control, according to an adaptable stochastic policy. In our parlance, a control corresponds to a decision D, so the agent is described by $P_{w}(D \mid \mathbf{X}(\mathbf{i}))$, where $w$ is an adaptable parameter. 
- On the next time step $n$, the process transitions to state $\mathbf{j}$ with probability $p_{\mathbf{i} \rightarrow \mathbf{j}}(D)$, where $D$ is the decision just made by the agent.

- The agent receives reward $R(\mathbf{j})$.

As a consequence of the decisions made, the agent thus receives a sequence of rewards $R\left(\mathbf{j}_{n}\right)$, and the goal of the agent is to maximize the long term average reward rate $r(w)$. Formally

$$
r(w)=\lim _{N \rightarrow \infty} \frac{1}{N}\left\langle\sum_{n=1}^{N} R\left(\mathbf{j}_{n}\right)\right\rangle
$$

where the angle brackets denote the expectation over the stochastic process.

Before presenting the gradient rule for maximizing $r(w)$, let us show how this framework applies to the learning problems studied in the main text. As example we consider the stimulus response association task when reward is delayed by $\Delta t=650 \mathrm{~ms}$, i.e. a bit longer than the $500 \mathrm{~ms}$ duration of a single stimulus. Then, at the onset of the presentation of $n$-th stimulus, the Markovian state $\mathbf{j}_{n}$ comprises the following three elements:

$\mathbf{j}_{n}^{S}$ : The pure version of the $n$-th stimulus.

$\mathbf{j}_{n}^{c_{1}}$ : A flag which is \pm 1 according to whether the decision in response to stimulus $n-1$ was correct.

$\mathbf{j}_{n}^{c_{2}}: \mathrm{A} \pm 1$ flag according to whether the response to stimulus $n-2$ was correct.

The partial observation $\mathbf{X}\left(\mathbf{j}_{n}\right)$ actually presented to the network is a jittered version of the stimulus $\mathbf{j}_{n}^{S}$. The reward $R\left(\mathbf{j}_{n}\right)$, delivered $150 \mathrm{~ms}$ into the presentation of stimulus $n$, is the value of $\mathbf{j}_{n}^{c_{2}}$. Once the network has responded to stimulus $n$, the flag $\mathbf{j}_{n+1}^{c_{1}}$ in the next state $\mathbf{j}_{n+1}$ is set according to the correctness of this response. Further, for $\mathbf{j}_{n+1}^{S}$ a next stimulus is picked at random and, finally, the $\mathbf{j}_{n+1}^{c_{2}}$ flag is set from $\mathbf{j}_{n}$ using $\mathbf{j}_{n+1}^{c_{2}}=\mathbf{j}_{n}^{c_{1}}$.

Baxter and Bartlett consider the following eligibility trace computed while sampling the decision process:

$$
e_{n+1}=(1-\gamma) e_{n}+\frac{\frac{\partial}{\partial w} P_{w}\left(D_{n} \mid \mathbf{X}\left(\mathbf{j}_{n}\right)\right)}{P_{w}\left(D_{n} \mid \mathbf{X}\left(\mathbf{j}_{n}\right)\right)}
$$

Here $0<\gamma<1$ is a discount factor and $D_{n}$ is the decision actually made in the $n$-th time step. They next introduce the reward weighted average of $e_{n}$,

$$
g_{n}=\frac{1}{n} \sum_{m=1}^{n} R\left(\mathbf{j}_{m}\right) e_{m}
$$

and relate $g_{n}$ to the gradient of a suitable objective function which we denote by $r_{\gamma}(w)$. In more detail, Baxter and Bartlett show that, under mild regularity conditions on the decision process

$$
\lim _{n \rightarrow \infty} g_{n}=\frac{\partial}{\partial w} r_{\gamma}(w) \quad \text { with probability } 1 \text {. }
$$

Of course, what we actually want to maximize is the average reward rate $r(w)$ and the relationship to the objective function is

$$
\lim _{\gamma \rightarrow 0} r_{\gamma}(w)=r(w)
$$

But to assure that $g_{n}$ has finite variance, we need to use a positive value of $\gamma$. So for the proposed procedure there is a bias-variance tradeoff in choosing the discount factor $\gamma$. In a companion paper 
(Ref. [2] main text), Baxter and Bartlett show that the above result leads to the following stochastic gradient procedure for the online adaption of $w$ :

$$
w_{n}-w_{n-1}=\eta_{n} R\left(j_{n}\right) e_{n}
$$

with positive learning rates $\eta_{n}$. Technically, to assure convergence, one has to assume that $\eta_{n}$ is not fixed in time but that it decays to 0 according to a suitable schedule. For biological systems this solution to the stability-plasticity dilemma seems unrealistic and, below, we shall stick to a fixed learning rate.

\section{Population learning for POMDP's}

To arrive at a first version of our population learning procedure we simply plug Eq. (3) in to Eq. (4)

$$
e_{n+1}=(1-\gamma) e_{n}+\int \mathrm{d} Y \mathrm{~d} A^{\backslash} P_{w}\left(A^{\backslash}, Y \mid D_{n}, \mathbf{X}_{n}\right) \frac{\mathrm{D} P\left(D_{n} \mid A^{\backslash}\right)}{P\left(D_{n} \mid A\right)} c(Y) \frac{\partial}{\partial w} \ln P_{w}\left(Y \mid \mathbf{X}_{n}\right)
$$

Here $\mathbf{X}_{n}$ is the stimulus presented at the $n$-th time step and in contrast to Eq. (3) we now make the stimulus dependence explicit in the notation. The fixed learning rate update simply is

$$
w_{n}-w_{n-1}=\eta R_{n} e_{n}
$$

As it stands the above procedure is an unhappy compromise. The averaging over stimuli, decisions and rewards is achieved by Monte-Carlo sampling during the evolution of the decision process, whereas the averaging over the variables $A \backslash$ and $Y$, internal to the decision network, is done separately for each time step in Eq. (5). Since, conditioned on the decisions $D_{n}$, the evolution of the process is independent of the internal variables, it is more natural to also leave the averaging over $A \backslash$ and $Y$ to the Monte-Carlo procedure. This amounts to simply using

$$
e_{n+1}=(1-\gamma) e_{n}+\frac{\mathrm{D} P\left(D_{n} \mid A_{n}^{\backslash}\right)}{P\left(D_{n} \mid A_{n}\right)} c\left(Y_{n}\right) \frac{\partial}{\partial w} \ln P_{w}\left(Y_{n} \mid \mathbf{X}_{n}\right)
$$

instead of Eq. (5). In first instance, the sampling prescription for the decision process now is to pick $D_{n}$ from $P_{w}\left(D_{n} \mid \mathbf{X}_{n}\right)$ and then pick $A_{n}^{\backslash}$ as well as $Y_{n}$ from $P_{w}\left(A_{n}^{\backslash}, Y_{n} \mid D_{n}, \mathbf{X}_{n}\right)$. But this just amounts to sampling the joint density $P_{w}\left(D_{n}, A_{n}^{\backslash}, Y_{n} \mid \mathbf{X}_{n}\right)$. The natural way to sample this joint density is to have the population generate spike trains in response to the stimulus, use this to calculate $A_{n}^{\backslash}$ as well as $A_{n}=A_{n}^{\backslash}+c(y) / \sqrt{N}$, and then sample $P\left(D_{n} \mid A_{n}\right)$.

The expression for $e_{n}$ can be simplified by assuming that the population size $N$ is large. We can then replace the finite difference in $\mathrm{DP}\left(D_{n} \mid A_{n}^{\backslash}\right)$, see Eq. 2, by a differential obtaining

$$
\begin{aligned}
\mathrm{DP}\left(D_{n} \mid A_{n}^{\backslash}\right) & =\frac{1}{\sqrt{N}} \frac{\partial}{\partial A_{n}^{\backslash}} P\left(D_{n} \mid A_{n}^{\backslash}\right)+\mathcal{O}(1 / N) \\
& =\frac{1}{\sqrt{N}} \frac{\partial}{\partial A_{n}} P\left(D_{n} \mid A_{n}\right)+\mathcal{O}(1 / N)
\end{aligned}
$$

where in the last line we have used that the difference between $A_{n}^{\backslash}$ and $A_{n}$ is $\mathcal{O}(1 / \sqrt{N})$. We use this for simplifying (6) to

$$
e_{n+1}=(1-\gamma) e_{n}+\mathcal{O}(1 / N)+\left(\frac{\partial}{\partial w} \ln P_{w}\left(Y_{n} \mid \mathbf{X}_{n}\right)\right) c\left(Y_{n}\right) \frac{1}{\sqrt{N}} \frac{\partial}{\partial A_{n}} \ln P\left(D_{n} \mid A_{n}\right) .
$$

We can now compare this to the last eligibility trace $E_{3}$ of the online cascade proposed in the main text

$$
\tau_{R} \dot{E}_{3}=-E_{3}+E_{2}(t) \operatorname{post}_{2}(t) \operatorname{Dec}(t)
$$


and observe the following correspondences between the increment in $E_{3}$ and in Eq. (7). As mentioned in Methods, $E_{2}$ is a low pass filtering approximation to $\frac{\partial}{\partial w} \ln P_{w}\left(Y_{n} \mid \mathbf{X}_{n}\right)$ and $\operatorname{post}_{2}(t)$ is the continuous encoding of $c\left(Y_{n}\right)$. For the specific decision circuitry used, $\frac{\partial}{\partial A_{n}} \ln P\left(D_{n} \mid A_{n}\right)=D_{n}-\tanh \left(A_{n}\right)$. The latter term provides the modulation of the decision feedback Dec $(t)$ given in Methods. For the online procedure, the $1 / \sqrt{N}$ factor in (7) is absorbed into the learning rate. Finally the discount factor $\gamma$ corresponds to the ratio of stimulus duration $T$ to $\tau_{R}$.

\section{References}

1. Baxter J, Bartlett P (2001) Infinite-horizon policy-gradient estimation. J Artif Intell Res 15: 319350.

2. Baxter J, Bartlett P, Weaver L (2001) Experiments with infinite-horizon, policy-gradient estimation. J Artif Intell Res 15: 351-381. 